\title{
Rao-Blackwellized Particle Filtering for 6-DOF Estimation of Attitude and Position via GPS and Inertial Sensors
}

\author{
Paul Vernaza and Daniel D. Lee \\ GRASP Lab, Department of Electrical and Systems Engineering \\ University of Pennsylvania, Philadelphia, PA 19104 \\ \{vernaza,ddlee\}@seas.upenn.edu
}

\begin{abstract}
The authors present an innovative method for the efficient joint estimation of attitude and position in six degrees of freedom via sensors such as GPS, inertial measurement units, and odometry. Traditional methods for attitude estimation via Kalman filtering are beset by many conceptual problems relating to the representation of orientations in linear spaces, leading to difficulties in implementation and the interpretation of uncertainty estimates, among other issues. These problems are compounded when it is necessary to jointly estimate position and attitude. We demonstrate how Rao-Blackwellized particle filtering provides a framework for approaching this estimation problem that is both conceptually appealing and practical. Results are shown that demonstrate the filter's robustness to sensor outages and its ability to perform well even in situations with noisy sensors and high initial uncertainty in all state dimensions; these situations are precisely those in which traditional Kalmanfiltering approaches are most likely to experience problems.
\end{abstract}

\section{INTRODUCTION}

We consider the problem of estimation of the six degrees of freedom (three translational and three rotational) that specify the pose of a vehicle in the world using primarily GPS, inertial, and odometric sensors. Such applications are becoming increasingly more common due to the ubiquity of global positioning system (GPS) receivers and the fairly recent development of low-cost inertial sensors based on micro electro-mechanical systems (MEMS) technology. In conjunction with simple odometry or comparable dynamic modeling, it is possible to assemble cheap sensor packages that provide all the information necessary for a vehicle to robustly localize itself. However, actual implementations of complete pose estimation systems are seemingly rare outside the unmanned aerial vehicle, mobile robotics, and aerospace communities. One possible reason for this is that despite the availability of suitable sensors, the design of estimators necessary to integrate sensor information in a probabilistic framework, is still a problematic task with no universallyaccepted solution. We will discuss in this paper some of the reasons why the task of 6-DOF vehicle pose estimator design is challenging, and we will propose a novel approach based on Rao-Blackwellized particle filtering that eschews some of the key problems associated with typical estimators while also admitting a fairly simple implementation that may be preferable over standard techniques for practitioners of pose estimator design.

\section{Previous Work}

Attitude estimation in itself is a subject that has been studied extensively by the aerospace community. State-space approaches have been employed for satellite applications since the mid 1960's [1], with Kalman filtering in its various incarnations being the preferred method. A typical approach involves applying an extended Kalman filter (EKF) to linearize the process and measurement models, which tend to involve highly nonlinear angular rate integrations in the process model and nonlinear coordinate frame transformations in the measurement model [2]. Innovations over the years include the use of quaternions as a nonsingular representation of orientations of minimal dimension, and the practice of estimating auxiliary parameters that represent deviations from a reference quaternion [3] [4]. More recent work has employed the sigma-point Kalman filter (SPKF) that removes the need for linearization while providing estimates that capture the statistics of the target distribution more accurately than the EKF [4] [5].

Although such approaches have proved adequate in certain applications, there are certain aspects of Kalman-filterbased attitude estimation that leave much to be desired in a theoretical sense. The representational issue is perhaps the most critical failing. Although some parameterizations of the three-dimensional group of rotations, $\mathrm{SO}(3)$, may superficially appear vector-like, there is in reality no one-to-one mapping of rotations to a vector space. The Kalman filter's assumption of linearity is thus violated in the most fundamental way. Not only are the dynamics of orientation estimation nonlinear, but the space of orientations itself is not a linear space. This implies that the component-wise addition of the parameters of two rotations is not a meaningful operation. As an example, the addition of two unit quaternions yields a quantity that is no longer a unit quaternion.

A related issue is the representation of attitudinal uncertainty. The most straightforward approach, treating the rotational parameters as random variables and defining an analytic distribution over them, is problematic due to the fact that the rotations lie in a nonlinear space. This can be seen in the case of attempting to define a Gaussian distribution over a onedimensional random rotation variable. We cannot restrict the range of valid angles to $[0,2 \pi)$, since a Gaussian cannot be 


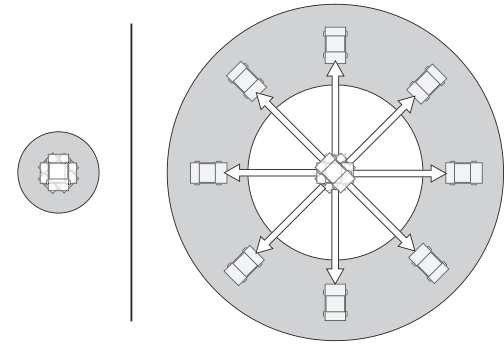

Fig. 1. Illustration depicting the multimodal nature of translational distributions that arise from common situations in vehicle pose estimation. Gray area represents range of possible vehicle locations. If orientation is unknown, given only an observation that the vehicle moves forward, the distribution over positions shifts from a unimodal (left) to a multimodal (right) distribution.

defined over a finite range; nor can we allow the angles to take on any real value, since this would lead to the assignment of an infinite number probabilities to each angle. Markley describes [3] how the use of an auxiliary parameter can be used to alleviate some of the conceptual problems with the direct estimation of quaternions by defining a distribution over a parameter that does lie in a linear space, and defining the true quaternion estimate as a function of this parameter. However, it is still not clear how a distribution over such parameters should map to the space of orientations in practical terms.

Yang and Crassidis have recently demonstrated how particle filtering may be applied to the problem of spacecraft attitude estimation [6]. We also employ particle filtering for attitude estimation in this work. However, we also demonstrate joint estimation of attitude and position in an efficient, unified framework.

Integrated state estimation of position and attitude has recently been examined by a number of researchers in the contexts of mobile robotics and especially autonomous helicopter flight. More often than not, the problem is decomposed into the two problems of first designing an orientation estimator and then constructing a separate, straightforward estimator for position given the best estimate of the orientation estimator. Such an approach is described by Roumeliotis et. al. [7]. This approach is appealing from an implementation standpoint, since it avoids some of the difficult issues that arise in attempting to estimate position and attitude states in a unified filter. However, it ignores the important structure of the correlation between attitudinal and positional uncertainty. Fig. (1) illustrates a common scenario where the correct understanding of the correlation between attitudinal and positional uncertainty is critical to inferences that might arise later.

A more integrated approach to simultaneous attitudinal and positional estimation is described by van der Merwe and Wan [8], who apply a SPKF to estimate a jointly Gaussian distribution over orientation and position for an unmanned aerial vehicle (UAV). The resulting filter is found to be more accurate than an EKF used for the same purpose. Unfortunately, the representation of the state by a single Gaussian is still a limiting factor. As Fig. (1) demonstrates, when passengers in a vehicle with unknown absolute orientation observe just forward motion, their marginal belief of absolute position becomes multimodal and hence is not adequately described by a single Gaussian. We would thus expect any estimator based on a single Gaussian assumption to fail on this example.

Our approach is based on a Rao-Blackwellized particle filter. The use of Rao-Blackwellized particle filters for integrated navigation tasks has been proposed independently by Nordlund [9] and Giremus et. al. [10]. We place specific emphasis on the advantages gained by sampling orientation, both theoretically and practically. We additionally demonstrate how these putative gains translate to practical advantages, especially robustness in highly uncertain scenarios, via experiments on an actual robotic platform.

\section{PRELIMINARIES}

It should first be made clear that we are approaching the problem from a strictly time-domain, state space view. This is in contrast to more classical frequency-domain approaches to filter design. Although some success has been achieved in applying complementary filters to attitude estimation (see Baerveldt for an example [11]), such approaches have problems of their own in addition to some of the ones already mentioned in state-space formulations. We will thus focus on state-space methods from this point forward.

As already described, standard methods for attitude estimation rely on assumptions of near-linearity and unimodality, and hence make use of nonlinear variants of the Kalman filter. It has already been shown that these assumptions are violated in some important cases that arise in vehicle pose estimation. Hence we are naturally drawn to particle filtering, a nonlinear estimation strategy that has experienced much success in the domain of mobile robot localization [12]. Particle filtering attempts to approximate the posterior state distribution by a finite weighted sum of localized delta functions, or "particles," as depicted in Eq. (1) [13], where the notation $x^{t}$ denotes the sequence $x_{1} \ldots x_{t}, w_{t}^{(i)}$ denotes the weight of the $i$ th particle, and $x^{(i)}$ denotes the value of that particle's state

$$
p\left(x^{t} \mid z^{t}\right) \approx \sum_{i=1}^{N} w_{t}^{(i)} \delta\left(x^{t}-\left(x^{(i)}\right)^{t}\right)
$$

Given this approximate representation it is then straightforward to calculate an approximation to the expectation of any function of the state [14]. Crucially, it can be shown that there exists a recursive method of selecting the weights such that the approximate expectation converges to the true expectation as the number of particles goes to infinity. Practically speaking, many cases require only a small number of particles for a satisfactory approximation.

The method of selecting the weights relies on a principle known as importance sampling, whereby we attempt to sample from a difficult-to-sample target distribution by first sampling from an easy-to-sample proposal distribution. We then obtain an approximate sampling of the target distribution by weighting the original samples according to Eq. (2) [15], and sampling from the discrete distribution of weighted samples. 


$$
w^{(i)}=\frac{\text { target distribution probability }}{\text { proposal distribution probability }}
$$

The correct calculation of the weights according to the principle of importance sampling is a critical step in the operation of the filter. We will later derive how to correctly set the weights for our application.

The recursive calculation of weights over time leads to a problem known as "particle degeneracy," [13] in which all but a few particles have habitually insignificant weights, meaning that they contribute little to the estimate and are a burden on computational resources. Thus nearly all practical particle filters implement a technique known as resampling to address this condition. Resampling consists of periodically "refreshing" the samples by sampling them from their own weighted distribution. We will not pay special attention to resampling here as we use a standard technique for it.

The final prerequisite for our development of the joint attitude and position estimator is the concept of "RaoBlackwellization." In the context of particle filters, this refers to the practice of exploiting the structure of certain state-space models where it is possible to analytically update part of the state when another part of the state is sampled. Fig. (2) is a graphical model that depicts the sort of conditional independence structure necessary to make Rao-Blackwellization applicable [16]. Where applicable, Rao-Blackwellization can often provide a better estimate of part of the state at a fraction of the computational cost of sampling the entire state. This is due to the fact that particle filtering becomes increasingly more difficult in high-dimensional state spaces, since the number of particles necessary to effectively sample the space grows as the volume of the sampled space grows.

An especially nice instantiation of the kind of model depicted in Fig. (2) arises when the distributions of $x_{t}$ and $z_{t}$ are Gaussian when conditioned on the sampled part of the state. This case is known as a conditionally linear-Gaussian model [16], and it is appealing because it allows us to optimally compute the posterior distribution over $x_{t}$ given each sampled $r_{t}$ using the Kalman filter. The joint distribution over $x_{t}$ and $r_{t}$ is thus represented by a combination of particles and their corresponding Gaussians, as expressed in Eq. (3)

$$
\begin{aligned}
p\left(x_{t}, r_{t} \mid z^{t}\right) & =\sum_{i} w^{(i)} \delta\left(r_{t}-r_{t}^{(i)}\right) p\left(x_{t} \mid r_{t}^{(i)}, z^{t}\right) \\
& =\sum_{i} w^{(i)} \delta\left(r_{t}-r_{t}^{(i)}\right) \mathcal{N}\left(x_{t} ; \hat{x}^{(i)}, \Sigma_{t}^{(i)}\right)
\end{aligned}
$$

\section{DeVelopment of THE FILTER}

\section{A. Orientation Estimation via Dead Reckoning}

We will first discuss what a "dead-reckoning" approach to attitude estimation would entail in order to develop some of the details relating to using inertial measurements for this purpose. Despite the name, we might actually use angular rate gyroscope measurements in a dead-reckoning approach. The reason for this is that dynamic modeling for attitude

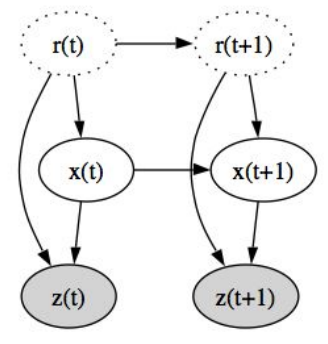

Fig. 2. A graphical model of the kind of dynamic Bayes network to which Rao-Blackwellization applies. We assume the state can be partitioned into $\mathrm{r}(\mathrm{t})$ and $\mathrm{x}(\mathrm{t})$ and that the conditional independence relations implied by the graph are present. $\mathrm{z}(\mathrm{t})$ is a measurement that may depend on both parts of the state.

estimation is application-specific, often complex, and usually yields results that are less accurate than those obtained from angular rate gyros, as described by Roumeliotis in [7]. We can thus think of angular rate gyros as providing a sort of control input to a process of integrating angular rates in time. This notion will be useful in our later development of a proper attitude estimator.

Another issue in the implementation of a simple deadreckoning approach is the choice of attitude representation. We will use quaternions in this work, though it will become apparent later that (unlike in traditional methods) our choice of representation has little impact on the design of the estimator.

The only significant operation in the dead-reckoning method is the repeated integration of angular rates over distinct time intervals to yield updated rotations. A closed-form solution of the appropriate differential equation is provided in [17] and is summarized in Eqs. (4) and (5), where $\omega_{t}=\left(\omega_{x} \omega_{y} \omega_{z}\right)$ is the angular velocity vector relative to the sensor's body frame, and $\mathbf{q}_{t}$ is a quaternion representing the sensor's orientation at time $t$.

$$
\begin{gathered}
\Omega\left(\omega_{t}, \Delta t\right)=\frac{\Delta t}{2}\left(\begin{array}{cccc}
0 & \omega_{z} & -\omega_{y} & \omega_{x} \\
-\omega_{z} & 0 & \omega_{x} & \omega_{y} \\
\omega_{y} & -\omega_{x} & 0 & \omega_{z} \\
-\omega_{x} & -\omega_{y} & -\omega_{z} & 0
\end{array}\right) \\
\mathbf{q}_{\mathbf{t}+\mathbf{1}}=e^{\Omega\left(\omega_{t}, \Delta t\right)} \mathbf{q}_{\mathbf{t}}
\end{gathered}
$$

\section{B. Orientation Estimation via Particle Filtering}

As alluded to earlier, we take a particle filtering approach to attitude estimation. By choosing a particle filter we immediately avoid most of the representational issues that plague Kalman-filter-based attitude estimators, since there is no need to define an analytical distribution over orientations. Instead, we will represent our orientation distribution by a set of discrete particles. This also has the advantage of providing a model of attitudinal uncertainty that has an extremely intuitive meaning compared to the artificial parameterizations present in some Kalman-filtering strategies. Intuitively, a highly uncertain distribution would be represented by a highly disordered set of orientations, while a very certain distribution would be represented by a tight cluster of orientations. 
Particle filter design is driven by the determination of the importance weights, which must be chosen in accordance with the principle of importance sampling. A straightforward derivation of the importance weights is given in Eq. (6) [13], where $q($.$) represents a yet-undetermined proposal density and$ $\theta$ represents the attitude.

$$
\begin{aligned}
w_{t}^{(i)} & =\frac{p\left(\theta^{t} \mid z^{t}\right)}{q\left(\theta^{t} \mid z^{t}\right)} \\
& =\frac{p\left(z_{t} \mid \theta^{t}, z^{t-1}\right) p\left(\theta_{t} \mid \theta_{t-1}\right) p\left(\theta^{t-1} \mid z^{t-1}\right)}{q\left(\theta_{t} \mid \theta^{t-1}, z^{t}\right) q\left(\theta^{t-1} \mid z^{t-1}\right)} \\
& =\frac{p\left(z_{t} \mid \theta^{t}, z^{t-1}\right) p\left(\theta_{t} \mid \theta_{t-1}\right)}{q\left(\theta_{t} \mid \theta^{t-1}, z^{t}\right)} w_{t-1}^{(i)}
\end{aligned}
$$

In the simplest form of the particle filter, we choose the state transition distribution $p\left(\theta_{t} \mid \theta_{t-1}\right)$ as the proposal density. The corresponding importance weights $w$ are then given by Eq. (7) [13]. Thus we only need to specify the state transition and observation likelihood distributions in order to apply the particle filter.

$$
w_{t}^{(i)}=p\left(z_{t} \mid \theta^{t}, z^{t-1}\right) w_{t-1}^{(i)}
$$

The state update is encapsulated in Eq. (8). Eq. (5) from the dead-reckoning approach essentially remains the quaternion update equation. Once again, the angular rate gyro is treated as a control input to the quaternion update process. $\omega_{t}^{\epsilon}$ represents error (assumed Gaussian) in the measurement from the rate gyro, $\omega_{t}^{g}$. Its inclusion ensures that repeated application of the process model results in an orientation distribution that grows more uncertain in time, as would be expected.

$$
\begin{aligned}
\mathbf{q}_{t+1} & =f_{q}\left(\mathbf{q}_{t}, \omega_{t}^{g}, \omega_{t}^{\epsilon}\right) \\
& =e^{\Omega\left(\omega_{t}^{g}+\omega_{t}^{\epsilon}, \Delta t\right)} \mathbf{q}_{t}
\end{aligned}
$$

Our measurements are given by body-referenced acceleration and possibly magnetic field. Assuming no external accelerations besides gravity or magnetic fields besides the earth's are present, Eqs. (9) and (10) are valid. They represent simply the rotation of their respective field vectors in fixed-frame coordinates $\left(\mathrm{g}^{f}\right.$ and $\left.\mathbf{m}^{f}\right)$ to the body-relative coordinates of the sensor frame, plus Gaussian measurement error $\mathbf{g}_{t}^{\epsilon}$ and $\mathbf{m}_{t}^{\epsilon}$. We assume that $R_{b 2 f}\{q\}$ is a function that returns a matrix transforming vectors in the body frame to the fixed (or "navigation") frame, given a quaternion. The precise form of it is omitted for brevity (see [17]).

$$
\begin{aligned}
\mathbf{g}_{t}^{b} & =o_{g}\left(\mathbf{q}_{t}, \mathbf{g}_{t}^{\epsilon}\right), \mathbf{g}_{t}^{\epsilon} \sim \mathcal{N}\left(0, C^{g}\right) \\
& =\left[\mathbf{R}_{\mathbf{f} \mathbf{2} \mathbf{b}}\left\{\mathbf{q}_{t}\right\}\right] \mathbf{g}^{f}+\mathbf{g}_{t}^{\epsilon} \\
\mathbf{m}_{t}^{b} & =o_{m}\left(\mathbf{q}_{t}, \mathbf{m}_{t}^{\epsilon}\right), \mathbf{m}_{t}^{\epsilon} \sim \mathcal{N}\left(0, C^{m}\right) \\
& =\left[\mathbf{R}_{\mathbf{f} \mathbf{2} \mathbf{b}}\left\{\mathbf{q}_{t}\right\}\right] \mathbf{m}^{f}+\mathbf{m}_{t}^{\epsilon}
\end{aligned}
$$

To calculate the importance weights, we must define the distribution $p\left(z_{t} \mid\left(\theta^{(i)}\right)^{t}, z^{t-1}\right)$. Given the models just described, one simple way to accomplish this is to define a Gaussian in the fixed frame as described in Eq. (11), where $v^{f}$ is either field vector in the fixed frame, and $R^{f}$ is the covariance of the measurement.

$$
p\left(z_{t} \mid \theta_{t}^{(i)}\right)=\mathcal{N}\left(z_{t} ; \mathbf{R}_{\mathbf{f} 2 \mathbf{b}}\left\{\mathbf{q}_{\mathbf{t}}^{(\mathbf{i})}\right\} \mathbf{v}^{f}, \mathbf{R}^{f}\right)
$$

A final important issue in the practical application of particle filtering to attitude estimation is the matter of how to calculate an "expected orientation" from a weighted set of sampled orientations. One procedure for accomplishing this is described by Morawiec in [18]. Briefly, a useful notion of mean orientation can be computed as the normalized principal eigenvector of the matrix $P$ shown in Eq. (12).

$$
\mathbf{P}=\sum_{i=1}^{N} w^{(i)}\left(\mathbf{4} \mathbf{q}^{(\mathbf{i})}\left(\mathbf{q}^{(\mathbf{i})}\right)^{\mathbf{T}}-\mathbf{I}_{\mathbf{4 \times 4}}\right)
$$

\section{Estimating Translation States via Rao-Blackwellization}

Our main idea in incorporating translational states into the filter will be to have particles representing orientations as before, but to also attach to each particle a Gaussian over positions. Rao-Blackwellization provides us with a principled way of accomplishing just that. An important observation is that most nonlinearities we might think of in the translational process or measurement models (utilizing sensors such as GPS and odometry) would tend to arise due to transformations between inertial and global coordinate systems, which involve products between translational states (or controls) and (possibly nonlinear) functions of attitude parameterizations. If the attitude were not part of the state, we could update the translational state with a standard Kalman filter.

We can solidify this intuition by using a graphical model. Looking at Fig. (2), it is apparent that the conditional independence structure depicted therein is applicable to our problem, where the translational state is represented by $x(t)$, and the sampled orientations are represented by $r(t)$. Furthermore, due to the observation that it is the orientation that produces nonlinearities in the translational dynamics, we have a conditionally linear-Gaussian model for the translational state. Therefore, Rao-Blackwellization is applicable, and we can use a linear Kalman filter to update the translational distributions conditioned on sampled orientations.

Knowing this, it now again necessary to examine how the importance weights should be calculated in the new model. When body-frame field measurements are observed, the model is essentially the same as it was in the case of orientation-only estimation, and hence the same update rules apply. In the case of GPS and odometric measurements, the model is different and will require accordingly different importance weights. The calculation of the weights depends on some features of the process and measurement models, so they are described in Eqs. (14)-(17). The state consists of three-dimensional position and velocity vectors in the fixed frame, as seen in (13).

$$
\mathbf{p}_{t}=\left(\begin{array}{llllll}
x_{t} & y_{t} & z_{t} & \dot{x}_{t} & \dot{y}_{t} & \dot{z}_{t}
\end{array}\right)^{T}
$$




$$
\begin{gathered}
\mathbf{p}_{\mathbf{t}+\mathbf{1}}=\mathbf{F}^{\mathbf{p o s}} \mathbf{p}_{\mathbf{t}}+\mathbf{v}_{\mathbf{t}}, \mathbf{v}_{\mathbf{t}} \sim \mathcal{N}\left(0, \mathbf{Q}^{\text {pos }}\right) \\
\mathbf{F}^{\text {pos }}=\left(\begin{array}{cc}
I_{3 x 3} & I_{3 x 3} \Delta T \\
0_{3 x 3} & I_{3 x 3}
\end{array}\right)
\end{gathered}
$$

Note that here we treat odometry readings as a measurement on body-relative velocity rather than a control input to update position. Eq. (16) expresses the GPS measurement as a direct measurement of the fixed-frame translational state, while Eq. (17) expresses odometry as a measurement of velocity rotated into the body frame.

$$
\begin{aligned}
& \mathbf{z}_{t}^{g p s}=\left(\begin{array}{ll}
I_{3 x 3} & 0_{3 x 3} \\
0_{3 x 3} & I_{3 x 3}
\end{array}\right) \mathbf{p}_{t}+\mathbf{w}^{g p s}, \mathbf{w}^{g p s} \sim \mathcal{N}\left(0, \mathbf{R}^{g p s}\right)
\end{aligned}
$$

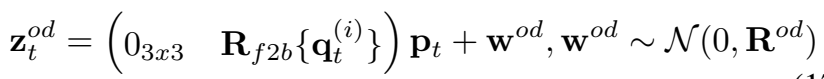

Eq. (17) demonstrates that, as anticipated, the odometry measurement is a linear function of the state when the orientation is given.

Given the process and measurement models, it is possible to calculate the importance weights for GPS and odometric measurements. We can again sample from the attitude update model to obtain a proposal distribution, so the importance weights are again as given by Eq. (7), since the same derivation as in Eq. (6) is applicable.

Though the form of the importance weights appears the same as the case with only attitudinal measurements, there are some important distinctions. Namely, the measurement equations for GPS and odometry are functions of position as well as attitude. We will therefore have to introduce the translational state by expressing the density in (7) as a marginal distribution over the translational state. Also note that since our conditional independence structure is now as specified in Fig. (2), $z_{t}$ and $z_{t-1}$ are no longer d-separated [19] by $\theta^{t}$, so we cannot immediately drop the $z^{t-1}$ term in the following derivation.

$$
\begin{aligned}
p\left(z_{t} \mid \theta^{t}, z^{t-1}\right) & =\int p\left(z_{t} \mid \theta^{t}, \mathbf{p}_{t}, z^{t-1}\right) p\left(\mathbf{p}_{t} \mid \theta^{t}, z^{t-1}\right) d \mathbf{p}_{t} \\
p\left(\mathbf{p}_{t} \mid \theta^{t}, z^{t-1}\right) & =\int p\left(\mathbf{p}_{t} \mid \mathbf{p}_{t-1}\right) p\left(\mathbf{p}_{t-1} \mid z^{t-1}, \theta^{t}\right) d \mathbf{p}_{t-1} \\
\boldsymbol{\Sigma}_{\mathbf{t}}^{-} & =\mathbf{F} \boldsymbol{\Sigma}_{\mathbf{t}-\mathbf{1}}^{(\mathbf{i})} \mathbf{F}^{\mathbf{T}}+\mathbf{Q}^{\mathbf{p o s}} \\
p\left(\mathbf{p}_{t} \mid \theta^{t}, z^{t-1}\right) & =\mathcal{N}\left(\mathbf{p}_{t} ; \mathbf{F} \hat{\mathbf{p}}_{t}^{(i)}, \boldsymbol{\Sigma}_{\mathbf{t}}^{-}\right) \\
p\left(z_{t} \mid \theta^{t}, z^{t-1}\right) & =\mathcal{N}\left(\mathbf{z}_{t} ; \mathbf{H F} \hat{\mathbf{p}}_{t}^{(i)}, \mathbf{H} \boldsymbol{\Sigma}_{\mathbf{t}}^{-} \mathbf{H}^{\mathbf{T}}+\mathbf{R}\right)
\end{aligned}
$$

In the above equations, $H$ and $R$ represent the appropriate measurement and measurement noise matrices obtained from either the GPS or odometric measurement models. Eqs. (19) and (20) are obtained by calculating the statistics of the relevant marginal distributions, which is easily accomplished given that the variables involved are linear functions of the translational prior state $p\left(\mathbf{p}_{t-1} \mid z^{t-1}\right)$, which is assumed to be distributed as a Gaussian with mean $\hat{p}_{t}^{(i)}$ and covariance $\Sigma_{t-1}^{(i)}$.

A fortunate characteristic of Eq. (20) is that the mean and covariance of the Gaussian distribution are already calculated when we use the Kalman filter to update the translational state distributions; hence, it is quite efficient to calculate the importance weights. We also note that Eq. (20) has the intuitive interpretation as being the probability of the actual measurement under the Gaussian that represents the expected measurement distribution given the estimated translational state distribution for each particle.

It is also worth mentioning that practical applications will usually desire simple one-vector summaries of the distribution. For this purpose we can easily compute the expected values for the orientation independent of position and vice-versa by marginalizing the joint distribution appropriately. It can be seen from Eq. (3) that this process yields a sum of weighted delta functions for orientations, and a mixture of Gaussians model for positions. The mean orientation may then be computed as already described, while the mean position is simply the weighted mean of the Gaussians. This was the technique employed to obtain the estimates used in the results sections.

The complete algorithm for joint attitude and position estimation is described in algorithm (1).

\section{Simulation Results}

We implemented the joint attitude and position filter and a simple simulator in order to test the filter's output in the case where the true trajectory was known. The simulations emphasize performance in scenarios with relatively high sensor noise, since it is expected that this is the area in which traditional implementations are most deficient. Gyro measurements, for example, were assumed to have a $0.1 \mathrm{rad} / \mathrm{s}$ Gaussian error in each dimension. Moreover, different scenarios were tested in which only certain sets of sensors were enabled in order to evaluate the filter's performance in conditions of sensor unavailability. None of the scenarios featured magnetometer measurements so as to render the absolute attitude unobservable in the static case. It is also crucially important to note that the yaw of the initial orientation was assumed to be completely unknown in each case. The filter was therefore initialized with a distribution of orientations having yaw angles uniformly distributed in the interval $[0,2 \pi)$. The true position and the filter's mean position estimate were both initialized to zero to enable comparisons between cases with and without GPS position measurements.

We ran the simulation in each scenario for 1000 seconds and calculated the error between the filter's output and the true state. The attitude error was computed as the minimum angle required to rotate the estimated orientation into the true orientation by rotating about an appropriate axis. The resulting plots of error versus time are provided in Fig. (3). A summary is provided in table (I), where $E|\tilde{x}|$ represents mean absolute error, and $\sqrt{E\left|\tilde{x}^{2}\right|}$ represents the standard deviation of the absolute error. 

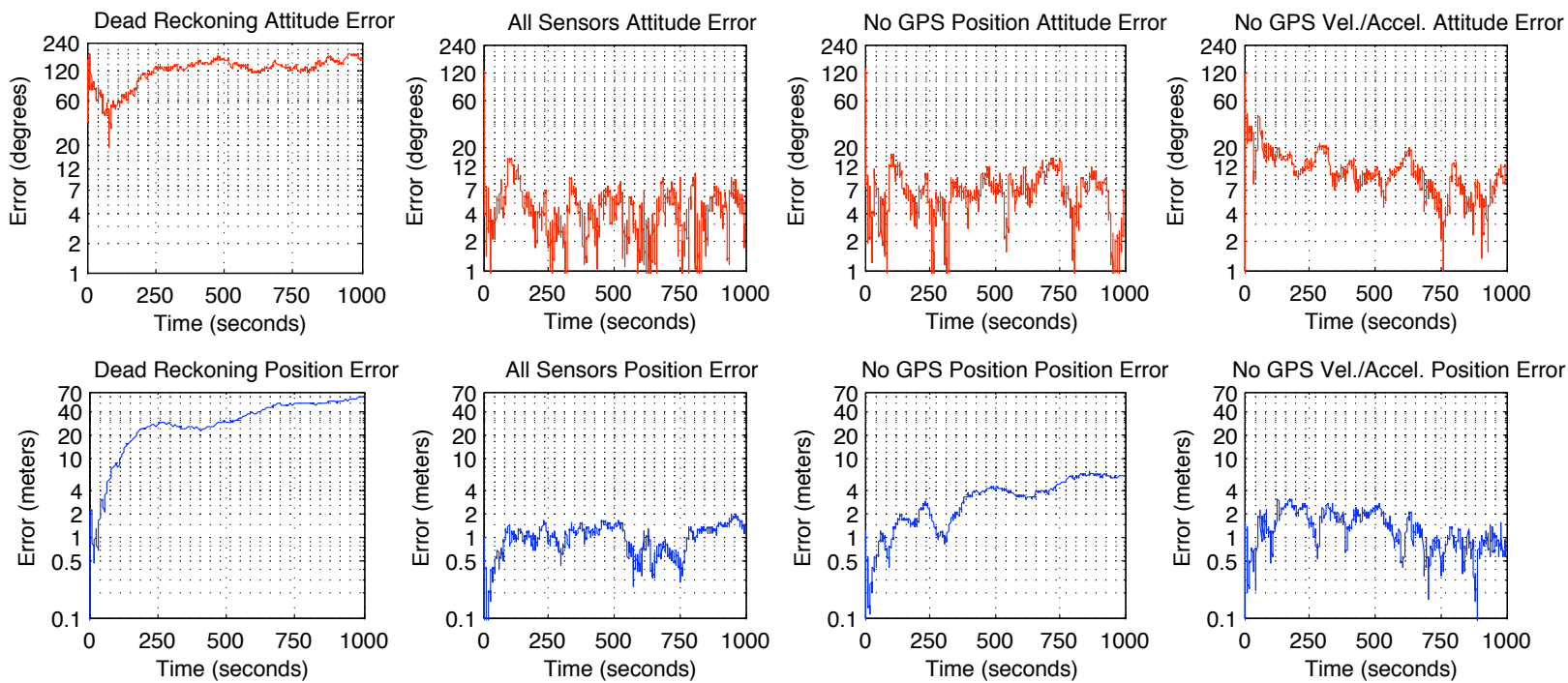

Fig. 3. Plots of simulation errors versus time for several scenarios (note log scale of error axis). A dead reckoning case (left) was provided for comparison. As expected, the best results were obtained with all sensors enabled. Disabling GPS position caused absolute position to slowly drift, but attitude error remained small. Disabling GPS velocity caused slower convergence, but both attitude and position error remained comparatively low in the long run.

A "dead-reckoning" case where the filter only employed rate gyro and odometry was included in the experiment to serve as a control. As expected, since the filter was given a uniform prior distribution over yaw angles and thereafter received nothing but relative measurements, the mean error in attitude remained very high throughout the simulation. The position error also remained high as a consequence of the orientation being unknown.

When all sensors were enabled, the filter was able to infer the correct orientation (with noisy sensors, from an unknown initial orientation) to within about five degrees on average. The absolute position error averaged about one meter. We consider this to be a good result considering that our simulated GPS consisted of random draws from a spherical Gaussian centered at the true position with a standard deviation of five meters, and obtained only once per second.

We then removed GPS position measurements completely, forcing the filter to rely on accurate attitude estimates and the integration of uncertain velocity. Though the attitude and position errors increased, the attitude and velocity estimates were good enough to result in a mean position error of 3.42 meters over the course of 1000 seconds.

Finally, we attempted the very difficult scenario of no GPS velocity or accelerometer measurements. In this case, the only way to infer the correct orientation is to essentially check the consistency of trajectories that arise from different initial orientations against the infrequent, noisy absolute position measurements provided by GPS. This was intended to be a test of the filter's probably unique ability to correctly estimate the complex, long-range correlations that arise between distributions over position and attitude. Although the mean orientation error in this case was nearly three times that of the case employing all sensors, the filter was able to resolve the orientation well enough to keep the error in the position estimate comparable to that of the case employing all sensors.

\section{Field Results}

We tested the filter's performance in a real-world, outdoor scenario by running it onboard a small, remote-controlled ground robot equipped with GPS, odometry, and an inertial measurement unit (IMU). As in the simulations, the magnetometer was not enabled, though this time for a more practical reason: as is often the case with small robots, local magnetic fields generated by the robot's motors make it difficult to sense the Earth's magnetic field. For this reason, in this application and similar ones, it is essential the the filter be able to resolve global orientation without the aid of a magnetometer. We therefore again gave the filter no initial knowledge as to the yaw component of the prior attitude distribution.

The test scenario consisted of driving the robot in a closedloop path around a relatively open field. The path was approximately 250 meters long. Reported GPS error averaged about 11 meters. The resulting 2D position tracks are displayed in Fig. (4). Although complete ground truth was not available for this experiment, we can make some arguments in favor of the conclusion that the filter is performing well.

The first observation we might make is that the filter is indeed successfully integrating low-frequency data from GPS with high-frequency gyroscope and odometry information to yield an estimate that is both detailed and driftless. The fact that absolute corrections are being made can be seen by observing that the estimate obtained without GPS position measurements does drift significantly in time compared to the estimate obtained using GPS position measurements. Closing the loop without GPS position measurements resulted in a 2.41 meter error at the end of the loop, while closing the loop with all sensors resulted in a 1.45 meter error. Raw GPS closed the loop with a 2.33 meter error. 


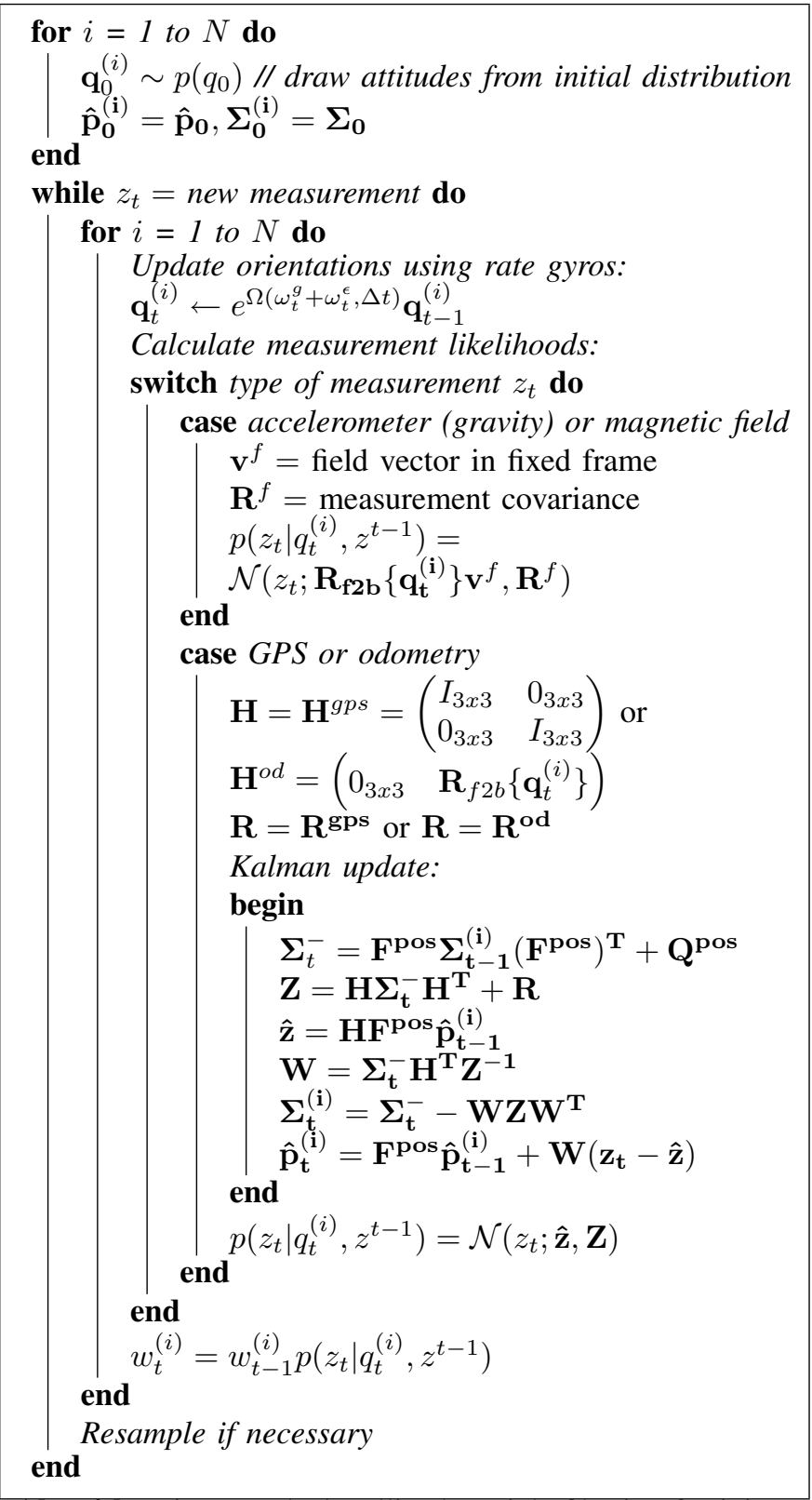

Algorithm 1: Rao-Blackwellized particle filtering for joint attitude and position estimation

\begin{tabular}{|l||l|l||l|l|}
\hline \multicolumn{1}{|c||}{} & \multicolumn{2}{c|}{ Attitude error (deg) } & \multicolumn{2}{c|}{ Position error (m) } \\
\hline Scenario & $\mathbf{E}|\tilde{x}|$ & $\sqrt{\mathbf{E}\left|\tilde{x}^{2}\right|}$ & $\mathbf{E}|\tilde{x}|$ & $\sqrt{\mathbf{E}\left|\tilde{x}^{2}\right|}$ \\
\hline \hline Dead-reckoning & 125.39 & 32.85 & 32.79 & 15.94 \\
All sensors (no & 4.86 & 3.98 & 1.04 & 0.39 \\
magnetometer) & & & & \\
No GPS position & 7.09 & 4.68 & 3.42 & 1.84 \\
No GPS & 11.82 & 6.71 & 1.40 & 0.70 \\
velocity or & & & & \\
accelerometers & & & & \\
\hline
\end{tabular}

TABLE I

SUMMARY OF SIMULATION RESULTS
Another important observation is that in all cases, the orientation was successfully resolved accurately and precisely from an initially unknown orientation. If this had not been the case, and the orientations had been inconsistent with absolute position and velocity measurements, the estimated tracks would have exhibited sharply jagged artifacts from constantly being "pushed" in one direction by odometry, and "pulled" in another via GPS. The degree to which the orientation was correctly inferred is evident in the loop-closing performance in the case without GPS position measurements, which indicated a drift of less than $1 \%$ over the length of the path traveled. Since, this figure is well within the error that might be expected from wheel slippage alone, we conclude that attitudinal error must have been fairly small throughout.

A final observation is that, as also demonstrated in simulation, the filter is able to accomplish the difficult task of convergence to a correct attitude even with very large initial uncertainty, using only GPS position measurements. This is illustrated by the dashed line in Fig. (4). Closing the loop in this case resulted in a 1.40 meter error. Since this is better than the loop-closing performance of raw GPS, it must be concluded that this is the result of the integration of correct orientation with odometry. However, it should be noted this result required a significantly greater number of particles for correct convergence than the other two, due to the initially larger space of feasible poses induced by this scenario.

Regarding computational efficiency, we should note that this is one area in which this filter is expected to lag a bit behind traditional implementations, since measurements on the translational state require a Kalman update for each particle, instead of a single Kalman update per measurement. Though this might seem like a greatly increased computational burden, it is alleviated by several factors. One very appealing aspect of the basic particle filtering algorithm is that it is almost completely parallelizable. Sampling the proposal density and calculating importance weights usually account for the vast majority of the computation burden, and both can be accomplished in parallel for each particle. The algorithm is also "any-time" in that the accuracy of the approximation is proportional to the number of particles employed, and techniques exist for adaptively choosing the number of particles such as to minimize computational cost while keeping approximation error low [20]. Practically speaking, we have found that even the most straightforward implementation produces adequate results with 100 particles and yields an average $20 \%$ CPU usage on a $2.0 \mathrm{GHz}$ Pentium M laptop. As few as 20 particles seems to produce reasonable results. More difficult cases featuring more complex distributions may require more. The experiment described above in which GPS velocity measurements were disabled was carried out with 300 particles, but still ran much faster than required for real-time operation on modest hardware. It is expected that a very good mix of accuracy and performance could be obtained by using adaptive techniques to effectively increase the number of particles during convergence and sensor dropouts and decrease them otherwise. 


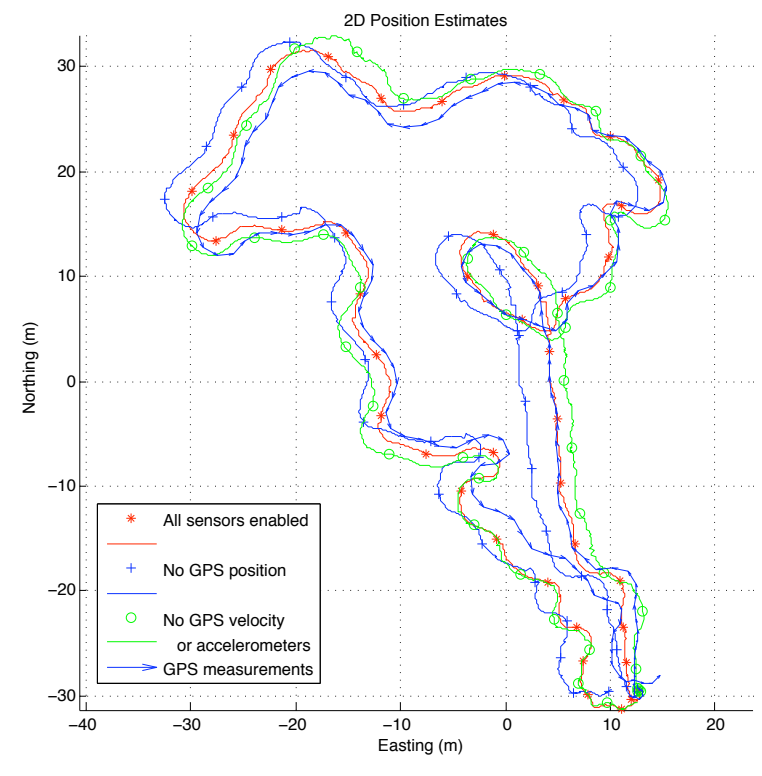

Fig. 4. Position estimates obtained by running pose filter on data obtained from real sensors. Arrowed line indicates location of GPS measurements. Line with asterisk markers indicates position estimates obtained by running filter with all sensors enabled. Line with plus markers indicates estimates obtained with GPS position measurements disabled shortly after initialization Line with circle markers indicates estimates obtained with GPS velocity and accelerometer measurements completely disabled.

\section{CONCLUSIONS}

We have presented a filter for joint attitude and position estimation of vehicles that combines the best features of currently-known techniques to yield an estimator that is efficient, accurate, and (in our opinion) attractive from both a theoretical and practical viewpoint. We achieved this by observing that many of the theoretical problems that arise in the estimation of attitude and joint attitude and position, can be avoided very simply by sampling orientations in a particle-filtering framework. We then demonstrated how RaoBlackwellization is very nicely applicable in the common case where nonlinearities in the process and measurement models arise principally from coordinate transformations mediated through the attitudinal part of the state. This leads to a filter that is very efficient, since the translational state distributions conditioned on the sampled orientations can be updated analytically using the Kalman filter.

Experiments with the filter in both simulation and real situations yielded performance that was good in both quantitative and qualitative aspects. Special consideration was given to cases with very noisy sensors, sensor unavailability, and highly uncertain initial pose distributions, where assumptions of linearity and normalcy relied on by standard approaches are most likely to fail. We demonstrated that the filter excels in all these cases, even managing to make the complex, long-range inference of orientation from very uncertain absolute position measurements.

In light of these results, we conclude that the applications that would most benefit from adoption of a filter such as this one would be those in which adequate computational power is available, sensors are relatively noisy, and a robust estimator is required. Such is often the case in mobile robotics. We have yet to study precisely how the filter compares to traditional approaches in cases where computational power is modest, sensors are significantly more accurate, uncertainties are smaller, and assumptions of linearity and normalcy might hold for practical purposes, such as is often the case in aerospace applications. However, it is hoped that future study will lead to applications in this domain as well.

\section{REFERENCES}

[1] I. Farrell, "Attitude determination by Kalman filtering," NASA, Contractor Report CR-598, 1966.

[2] J. Marins, X. Yun, E. Bachmann, R. McGhee, and M. Zyda, "An extended Kalman filter for quaternion-based orientation estimation using MARG sensors," in IEEE International Conference on Intelligent Robots and Systems, 2001.

[3] F. L. Markley, "Multiplicative vs. additive filtering for spacecraft attitude determination," in Dynamics and Control of Systems and Structures in Space, 2004.

[4] E. Kraft, "A quaternion-based unscented Kalman filter for orientation tracking," in Proceedings of the Sixth International Conference on Information Fusion, 2003.

[5] J. Crassidis and F. L. Markley, "Unscented filtering for spacecraft attitude estimation," Journal of Guidance, Control, and Dynamics, 2003.

[6] Y. Cheng and J. L. Crassidis, "Particle filtering for sequential spacecraft attitude estimation," in Collection of Technical Papers - AIAA Guidance, Navigation, and Control Conference, 2004.

[7] S. Roumeliotis, G. Sukhatme, and G. Bekey, "Circumventing dynamic modeling: Evaluation of the error-state Kalman filter applied to mobile robot localization," in Proceedings 1999 IEEE International Conference on Robotics and Automation, 1999.

[8] R. van der Merwe and E. Wan, "Sigma-point Kalman filters for integrated navigation," in 60th Annual Meeting of the Institute of Navigation, 2004.

[9] P.-J. Nordlund and F. Gustafsson, "Sequential Monte Carlo filtering techniques applied to integrated navigation systems," in Proceedings of the American Control Conference, 2001.

[10] A. Giremus, A. Doucet, V. Calmettes, and J.-Y. Tourneret, "A RaoBlackwellized particle filter for INS/GPS integration," in Proceedings of the IEEE International Conference on Acoustics, Speech, and Signal Processing, 2004.

[11] A.-J. Baerveldt and R. Klang, "A low-cost and low-weight attitude estimation system for an autonomous helicopter," in Proceedings of IEEE International Conference on Intelligent Engineering Systems, 1997.

[12] D. Fox, S. Thrun, W. Burgard, and F. Dellaert, "Particle filters for mobile robot localization," in Sequential Monte Carlo Methods in Practice. Springer-Verlag, 2001.

[13] S. Arulampalam, S. Maskell, N. Gordon, and T. Clapp, "A tutorial on particle filters for on-line non-linear/non-Gaussian Bayesian tracking," IEEE Transactions of Signal Processing, vol. 50, pp. 174-188, 2002.

[14] A. Doucet, "On sequential simulation-based methods for Bayesian filtering," Statistics and Computing, 2000.

[15] S. Thrun, M. Montemerlo, D. Koller, B. Wegbreit, J. Nieto, and E. Nebot, "FastSLAM: An efficient solution to the simultaneous localization and mapping problem with unknown data association," Journal of Machine Learning Research, 2004, to appear.

[16] K. Murphy and S. Russell, "Rao-Blackwellised particle filtering for dynamic Bayesian networks," in Sequential Monte Carlo Methods in Practice, 2001.

[17] J. Farrell and M. Barth, The Global Positioning System \& Inertial Navigation. McGraw-Hill, 1999.

[18] A. Morawiec, "A note on mean orientation," Journal of Applied Crystallography, October 1998.

[19] S. Russell and P. Norvig, Artificial Intelligence: A Modern Approach. Prentice Hall, 2002.

[20] D. Fox, "Adapting the sample size in particle filters through KLDsampling," International Journal of Robotics Research, 2003. 